\title{
Vorwort zur 107. Auflage
}

Der „Küster-Thiel“ bleibt auch im Zeitalter von Laptop, Google, Excel-Charts und sonstigen elektronischen Hilfen und Auswertverfahren ein unentbehrlicher Helfer in vielen Berufszweigen. In der vorliegenden Auflage wurden neben allgemeinen Korrekturen insbesondere die Atommassen der Elemente den aktuellen IUPAC-Daten angeglichen. Das Kapitel „Arbeitssicherheit“ wurde grundlegend überarbeitet, insbesondere wurde hier auf die neuen Systeme GHS/CLP zur Einstufung und Kennzeichnung von Chemikalien hingewiesen. Die sehr positive Resonanz auf Rechenbeispiele wurde zum Anlass genommen, weitere Beispielrechnungen hinzuzufügen.

Für die zahlreichen Anregungen und Hinweise möchten wir uns an dieser Stelle ganz herzlich bedanken.

Altenbach, April 2011

Alfred Ruland

Ursula Ruland 